Beyond robotic speech: mutual benefits to cognitive psychology and artificial intelligence from the study of multimodal communication

\author{
Beata J. Grzyb, Gabriella Vigliocco \\ $U C L$
}

oxford_regular30-eps-converted-to.pdf 



\section{Beyond robotic speech: mutual benefits to cognitive psychology and artificial intelligence from the study of multimodal communication}

\subsection{Introduction}

Language has predominantly been studied as a unimodal phenomenon (i.e. speech or text) without much consideration for the physical and social context in which communication takes place. In psychology, the study of language learning, production and comprehension has largely ignored the face-to-face communicative context in which language is learnt and processed, following a long-standing tradition in Linguistics according to which only the linguistic content is "language proper" (see [110] for a discussion). Thus, language has been defined as a rule-governed system which consists of abstract units (phonological, morphological, lexical) that can be separated from other aspects of communicative behaviour, such as gestures, gaze, prosody and the mouth movements associated with speech. These cues, although omnipresent in face-to-face context, are typically considered as "non-verbal" or "non-linguistic" and studied separately as part of non-verbal behaviour. There is, however, now a growing body of literature emphasizing the role of such cues in language learning and processing. For example, gestures such as pointing and iconic gestures (that imagistically evoke aspects of referents) have long been shown to have a powerful effect on language learning, predicting the onset of learning milestones [96]; they support speakers in organizing their thoughts for speaking [61] and help listeners, especially in noisy environments $[32]$.

Implementation of language in embodied agents (i.e. artificial agents that interact with their environment through the body including virtual agents and physical robots) has also been reduced to speech only (see [21] for overview and alternative approaches). This is problematic because in interactions with humans, the embodied agent is not capable of taking advantage of the wealth of cues produced by the speaker when comprehending and cannot provide these useful cues when producing language. Imagine for example a robot assisting an elderly person. The person asks her to bring him THAT glass (pointing toward the glass he wants). The robot has no way of understanding this indexical expression. As another example, imagine a robot and a human working on a joint task in a factory. The environment is noisy and the person has problems understanding what the robot says, the conversation is constantly interrupted and 
the person is frustrated. In human-to-human interactions, mouth movements, gesture and gaze can successfully be used to fill in for missed speech content, but this is not possible with a robot assistant. These examples illustrate why multimodality in human-machine interactions is desirable. As embodied agents, and especially robots, become more common in our living environments (e.g. virtual receptionists and tour guides, lab demonstrators or tutors, assistive robots in home, companion robots for the elderly), they will need to be endowed with face-to-face communication skills; especially in the settings where they have to interact with naïve users. The existing works on effects of robot multimodal behaviour on human-robot interaction have indicated that multimodality does increase the intuitiveness and naturalness [53], as well as the effectiveness and robustness of interaction [16,93], and finally, it positively influences the evaluation of agents increasing the chances of these agents to be used and accepted by their users $[66,13,100]$.

Both producing multimodal cues by embodied agents and detecting and understanding these cues in human users are complex problems. Embodied agents have to display cues in appropriate contexts, congruently with speech, and importantly, their responses need to vary. Otherwise their behavior is unbelievable, and embodied agents rapidly become rigid and unappealing. One limitation of cue generation in physical robots (but not in virtual agents) is the fine control of the movements where physical motors are used to control the appearance and timing of the movements. But more generally, a key limitation is our lack of knowledge of the underlying mechanisms of multimodal language production and comprehension in humans. Indeed, equipping embodied agents with multimodal language requires a detailed understanding of operational models of how the different cues are dynamically assembled and produced in humans.

In this paper, we advocate that artificial intelligence should take a more comprehensive stance on language to improve the effectiveness, intuitiveness and natural flow of human-machine interactions. In turn, computational methods can support collection and coding of human face-to-face communication data essential for developing artificial systems. Finally, the computational models that produce language in artificial embodied agents can provide a unique research paradigm for investigating the underlying mechanisms that govern language processing and learning in humans. Such a joint approach to the study of multimodal language will be beneficial for psychology/linguistics and artificial intelligence.

Below, we review the literature on the use of multimodal cues in human-to-human communication. We move next to discussing whether - and if so how - humans respond to multimodal cues displayed by embodied agents. Finally, we review how embodied agents can recognize multimodal cues, and how they can produce them. We close discussing the potential benefits to psychologists and computer scientists of joining forces in the study of multimodal communication.

\subsection{The use of multimodal cues in human face-to-face communication}

In face-to-face communication, the unfolding speech signal, which carries phonological, syntactic, semantic and discourse information, is accompanied by a multiplex of cues 
that recruit different articulators (manual, gaze, mouth patterns and vocal) ${ }^{1}$. These cues have all been argued to play an important role in adults' language processing and in children's word learning.

Iconic gestures evoke visual features of the referent (e.g. when describing a room, the speaker can represent the length of a piece of furniture by placing the hands far from each other) as well as properties of actions (e.g. when talking about a recipe, the movement of the hands can act out whisking an omelet). These gestures facilitate comprehension when the message they convey is congruent with speech, however, they can also impede comprehension when the message diverges from speech [57]. Furthermore, iconic gestures can help listeners to disambiguate the meaning of words (e.g., the word ball presented with a gesture congruent either with the dance or game meaning) [48].

Iconic gestures are important not only for the listener but also for the speaker. Individuals who gesture tend to speak more rapidly [5]. In contrast, the prohibition of gestures in narrations makes speech less fluent and causes a higher proportion of filled pauses [92]. Gesture production may facilitate speech formulation processes through decreasing cognitive load [41], priming conceptual information [67], activating information or maintaining the activation of spatial information [38, 98], and finally, preparing and structuring information for processing [60]. Furthermore, iconic gestures permit the speaker to express thoughts/concepts that otherwise would be difficult, if not impossible, to be expressed using speech only (e.g. describing the aspects of the coastline) [40]. Finally, gestures can also be used to depict more abstract concepts (e.g. mathematical notions etc., [4]).

Points (i.e. hand actions performed with an extended index finger or with other configurations of the hand) are used to focus their listener's attention on something, for instance, to refer to an object or to isolate the object from other objects in a complex scene. They are the most common multimodal cues used by caregivers [55, 84], and can facilitate language learning by helping to link a provided label with the referent.

Finally, object manipulations (e.g. showing a toy hammer to a child or showing how to use the toy hammer) can be used by speakers in a purely indexical manner (showing the object), or to order to communicate something about the object.

Iconic gestures, points, and object manipulations (i.e. manual cues) have been argued to support language learning by directing attention to specific objects present in the environment, or by bringing to the mind's eye properties of objects absent in the environment [111]. Iconic gestures and points are present early in in parental input to children [97], as well as in the gestural repertoires of children [1]. Importantly, it has been shown that parents' gesture use (specifically points) predicts children's gesture use, which in turn predicts later vocabulary development [97], indicating the importance of this cue for overall language development. A link between object manipulation

\footnotetext{
${ }^{1}$ The cues we focus on in this paper do not exhaust the repertoire of multimodal cues available in face-to-face communication as we are not including other aspects such as facial expression and head-body motion which also provide valid cues for learning and processing. The reason for limiting our attention to the cues described above is related to the fact that these have received the greatest attention in psychology.
} 
and children's vocabulary learning has also been suggested [94], while iconic gestures may not be present until somewhat later (around 28 months) when they are seen in parental input especially when objects are not present in the environment [111].

Gaze can be used by listeners to anticipate, ground and disambiguate spoken referents [105]. Speakers fixate on objects or locations in the immediate surrounding, one second or less before naming them [43, 44, 120]. Speaker's gaze can be used by listeners to identify the reference and to predict what the speaker is going to say. Indeed, listeners are slower at responding to the speaker's referential utterance when their access to the speaker's gaze is limited [15]. Gaze helps listeners to eliminate uncertainty and to disambiguate between referents $[44,105]$. In addition, gaze can signal communicative intent, and hence, modulate how listeners process speech and gestures [50]. Similar to the manual cues, gaze is an important cue from a very young age [109, 115]. Even young children systematically use the speaker's gaze to disambiguate the referent [10]. More generally a strong link between gaze behavior (i.e., gaze following, joint attention) and language development has been argued for $[75,18]$. For instance, gaze-following behavior at 10-11 months accompanied by vocalizations has been shown to predict language comprehension and gesture production at 18 months [18]. Gaze is often considered together with pointing as establishing joint attention. However, when in conflict, the two cues dissociate in their developmental trajectory with gaze providing the strongest cue earlier on (about 14 months) and pointing becoming the preferred cue later on (by 24 months) [87].

Face-to-face communication allows listeners to make use of mouth movements that accompany speech. Interestingly, dubbing a voice saying $[\mathrm{b}]$ onto a face articulating $[\mathrm{g}]$ results in hearing [d]. This is an example of a powerful "McGurk illusion" [74], which has been shown both in adults and in infants [20, 68]. In more naturalistic presentations of the audio-visual speech, it is well established that mouth movements facilitate speech perception by reducing lexical competition, especially in noisy conditions (for a review, see [88]). They can also speed up speech perception $[25,114]$ by providing predictive information about forthcoming phonemes. Finally, mouth movements were suggested to directly and automatically influence speech comprehension [9].

In addition to visual cues, the prosodic contour of the speech provides meaningful information to listeners. While prosody is often considered as part of the linguistic information provided in speech, in most psycholinguistic research, prosodic information is reduced to achieve experimental control. However, prosody is used by the speakers to draw listeners' attention to words that introduce new information [14], it can be iconic (e.g, referring to a long trip by saying looong), and it can provide important cues to word boundaries (see review in [28]). Prosody (especially changes in pitch) has long been recognized as a key property of child-directed speech [34], and is helpful in word segmentation [107], syntactic processing [45] and word learning more generally $[46,42,71]$.

These multimodal cues can be classified in terms of whether they support referent mapping/disambiguation: for example, points, gaze and object manipulations all direct attention to referents present in the environment (they are indexical) and therefore they help singling the referent out. Iconic gestures and iconic prosody by virtue of 
being iconic can bring to the mind's eye properties of absent referents. They can also be classified in terms of whether they support semantic processing of the speech, such as iconic gestures, points, gaze and prosody (iconic prosody and the use of pitch/duration to mark new information), or whether they support sensory processing of the speech (mouth movements). Both classifications emphasize the supportive role of multimodal cues in language learning and use.

\subsection{How humans react to embodied agents that use multimodal cues?}

Human-machine interaction focuses on understanding how the different features of an embodied agent (i.e., how the robot looks like or behaves) affect its perception by the human in terms of, for instance, agent's intelligibility and likeability, as well as effectiveness of interaction. Here, studies generally use 'Wizard-of-Oz' techniques (where a person manually chooses the agents output) or template-based techniques or prerecorded behaviours (i.e., speech, gestures etc.) without implementing these complex behaviours on an agent.

Because embodied agents, either physical robots or virtual agents, usually have some human-like features (e.g. human-like bodies, or body parts) naïve users expect them to display the whole range of human-like communicative behaviors. Human participants are sensitive to points and gaze displayed by a robot, and their ability to read robot's cues is aligned with their ability to read and interpret the same cues from other humans [16]. Robot gaze can successfully direct attention to object reference [2], as well as manage conversational turn-taking [6]. Similarly, human users can exploit robot's gaze to anticipate and disambiguate spoken references [105], and they adapt their behavior in response to the robot's gaze $[2,118]$. For instance, human participants looked more to a robot's face when the robot produced more looks to the person's face, thus creating more opportunities for mutual gaze and eye contact between the two [118].

Human-machine interactions are rated as more natural and intuitive when the agent uses a variety of co-speech cues. For instance, Iio et al. [53] showed that human participants rated higher the naturalness and ease of instruction and understanding of a robot that used gaze and pointing in addition to speech as opposed to a robot who used speech and gaze only or speech and pointing only; showing advantages of using multimodal channels for communication. Similarly, embodied agents that produced a range of different gestures were evaluated as more natural than agents that only produced points [73]. In addition, agents that produce communicative gestures such as movement of the eyebrows, or non-communicative such as body scratching are perceived as more lifelike [66], as well as more expressive, enjoyable and having positive personality [19]. Similarly, robot gaze was shown to increase the perception of robot human-likeness, and increase the positive evaluation of the agents [56, 47].

There is evidence that multimodal cues can also improve the effectiveness of humanmachine communication. In the study of Breazeal et al. [16], naïve human participants guided a robot to perform a physical task using both speech and gesture. In one case, the robot pro-actively communicated its internal states both implicitly through gaze cue (e.g. where the robot looks and when the robot makes eye contact with 
the human participant) and explicitly using other communicative cues (e.g. nods of the head, points, facial expressions). In the other case, the robot communicated only when prompted by the human and using only explicit cues that do not show the robot's internal state (e.g., only looking straight ahead; no expressions of confusions, and only responds with head nods and shakes when prompted by the human). The results of a questionnaire and behavioural analysis of video revealed that participants could better understand the robot's current state and abilities when the robot was displaying implicit cues. The communication with such a robot was also more efficient and resistant to errors. As in case of gestures, gaze behavior also improved the agent's evaluations in cooperative tasks [15].

While multimodal cues have a general positive effect in embodied agents, their timing and congruency with the speech is key. Just as in human agents, gestures that are incongruent with the speech have a negative effect on comprehension of embodied agents. For example, in Bergmann, Kopp Eyssel's study [13], an embodied agent with no gestures was rated more positively as compared with an agent that randomly produced gestures. Moreover, the ratings of the agent with random gestures were lower for overall comprehension, as well as comprehension of iconic gestures, and the certainty of the agent's communicative intentions; and the incongruent gestures displayed by the robot negatively contributed the participants' task-related performance [100].

\subsection{Can embodied agents recognize multimodal cues produced by humans?}

Recognition of multimodal cues produced by agents is a prerequisite to using them to improve comprehension by embodied agents especially in situations in which speech recognition is not optimal, like noisy environments (as most of every-day settings). Recognition of multimodal cues is hard for artificial systems. The existing robotic systems can only recognize a few, usually pre-defined, hand gestures. For instance, a system proposed by Xiao et al. [117] can recognize a set of 12 communicative gestures and actions with objects, while a system based on a deep neural network can robustly recognize 5 gestures performed by different people [11]. More recently, Castillo et al. [24] proposed a system able to recognize 14 different gestures (i.e., come toward, stop, greeting, pointing left/right). Only few attempts focused on recognition of iconic gestures [103, 62], and these were able to recognize gestures, iconic of a few objects, based on the similarity between the shape of the object and the shape of the hand or its motion. Recognizing pointing gestures is easier for embodied agents - given a welldefined and unique hand posture - and existing systems achieve good accuracy $[82,33]$. For instance, the system devised by Nickel and Stiefelhagen [82] can detect pointing (with $88 \%$ success rate) as well as appropriately indicate the target of pointing (one of eight objects).

A number of studies addressed the problem of detecting gaze direction in human users in order to drive the behaviour of embodied agents. These systems often use the direction of head as an approximation of gaze [31, 54]. As an alternative, some studies estimate the gaze direction either using head-mounted [118] or remote eye-trackers [85]. Detection/recognition of objects at which the human user is looking at, however, 
is difficult, especially in a naturalistic cluttered environment, and the systems usually are limited to a few objects.

Little attention has been paid to prosodic cues in the existing literature. Formolo and Bosse [37] extract prosodic features with the main purpose of detection of different emotions, Rosis et al. [95] looked at social attitude; Ang et al. [8] at frustration and annoyance.

Why recognition of multimodal cues is so difficult for embodied agents? One reason is the type of sensors that the robots or virtual agents use. For instance, due to low resolution of cameras used in robotics, the systems often use the direction of head as an approximation of gaze [31, 54]. However, in naturalistic setting people often make short gazes at objects without moving their heads. Systems that only use head direction are not sensitive to detecting such gazes. As an alternative, some robotics systems use head-mounted eye trackers [118]. However, using external devices prevents the naturalness of the human-machine interaction. In addition, changes in illumination can affect recognition of hand gestures based on visual input, and some studies use wearable gloves, or Microsoft Kinect to capture user's movement.

Another reason is that often the hand gesture recognition problem is limited to a pattern classification problem, in which the given input data (e.g. hand motion trajectory) is classified into one of few predefined categories. Although such an approach works well for recognizing points or predefined gestures (commands that a computer or a robot can understand), it is unsuitable for recognition of other meaningful (iconic) gestures which have no one-to-one mapping between their form and meaning (i.e., many gestures may have the same meaning or alternatively one gesture could have a different meaning, depending on the context).

Crucially, artificial systems usually rely only on a single modality for recognition (e.g. vision or motion) which is error-prone, due to background noise and changing light conditions, in real-word environments. Most often, these systems do not take advantage of other available cues that facilitate recognition. It is however the case that some existing studies show that multimodality improves recognition performance. For instance, the ICONIC gesture recognition system [63, 62] exploits the fact that most gestures co-occur with speech. More specifically, the system scans speech for key words that indicate a possibility of a gesture (e.g,, "like this") and once such a key word is detected, the system looks for a relevant gesture that accompanies the speech. Recognition of gestures can also be improved with help of prosodic information. Kettebekov et al. [58] investigated how the intonationally prominent parts of speech align with kinematically defined gesture primitives. The results of the analysis were then used to discard the falsely detected points as well as preparation gesture primitives.

Using speech, also improves detection of points and importantly of their referents. For instance, Showers and Si [102] showed that the accuracy of point and referent detection is higher when a robotic system uses a contextual information such as speech (extracted object names, object properties, and object position) and confidence heuristics (the quality of speech or visual information) as compared to a visual information alone. The same is true for gaze. Morency and Darrell [76] showed that using three types of contextual features such as lexical features, prosody/punctuation, and timing improves significantly the recognition of gaze. 
Although not used explicitly in embodied agents, mouth movements are often used in computational models of speech recognition. For instance, Bregler and Konig [17] showed that, in the presence of additive noise and crosstalk, such a combined architecture achieves better recognition rates than speech-only architecture. Other systems based on audio-visual (extracted visual information about the lip features) integration show an improvement of speech recognition in normal but also in noisy real-word environments. For instance, Noda et al. [83] proposed a system that combines a deep denoising autoencoder with a visual feature extraction mechanism that predicts the phonemes from the image sequence of the mouth area. They showed that using mouth information further improves the system performance (results in a lower error rate of word recognition).

Some studies attempted to improve the recognition of naturalistic conversational speech by utilizing information from deictic cues such as points and gaze. The object reference ambiguities in the system of Holzapfel, Nickel, Stielhagen [51] are resolved using either speech or through the interpretation of pointing. In addition, gaze can improve automatic speech recognition systems by highlighting the subset of vocabulary that relates to a person's visual attention. For instance, Cook et al. [27] proposed a model, which selectively uses the information from gaze (i.e. depending on the detected gaze role), and showed that under varying noise conditions, such a system achieves lower word error recognition rates. Finally, there have been some attempts to use prosody to aid automatic speech recognition. Here Fu et al. [39] show a decrease in word error rate when recognition network uses not only previous words but also prosodic features as an input.

\subsection{Can embodied agents produce multimodal cues?}

Humans produce a wide range of multimodal cues that are tightly linked to and timealigned with the accompanying speech. These cues have been shown both to facilitate language processing in listeners and to facilitate language production in speakers. We already know that human users are sensitive to the cues produced by embodied agents, but can embodied agents produce them?

Few studies have proposed computational systems that provide embodied agents with the ability to produce speech and co-speech gestures. Kopp and Wachsmuth [65] proposed a system that produces gestures from (manually) annotated descriptions which comprise speech transcript as well as gesture types (hand shape, palm orientation, hand location etc). Contrary to this model, the BEAT system [23] was able to infer from input text what gestures to produce and when. The system was provided with a "knowledge base" including information about objects, actions as well as gestures. In order to decide what gestures to use for a given text, the system first performs semantic analysis of the text marking novel words, and then it applies a set of rules derived from studies of human communication. For instance, it produces iconic gestures when during the explanation part of the utterance an object name is uttered and when this object has (as per knowledge base) some unusual features. Similarly, iconic gestures are produced for any action for which knowledge based contained a gesture description. Ng-hing, Luo, Okita [108] proposed instead a system that analyzed input text in order to produce co-speech gestures. It used an automatic tagger to determine 
for each word of input text the part of speech of the word and a text-to-speech engine to extract word timing information. Then, input text together with any automatically extracted tags was processed by several grammars, one for each gesture type, and several candidate gestures for each word of the text were proposed.

One main drawback of these gesture-production systems is that gestures are highly deterministic $[65,23]$ meaning that the exact same gestures are produced for the same input text. One way to solve this problem is to select gestures probabilistically, from the range of possible candidates, as it has been done by $\mathrm{Ng}$-hing, Luo, Okita [108] or to learn the probabilistic distributions of speech and gestures from a multimodal data collected from human participants [59, 12]. Such an approach was followed by Neff et al. [59], who derived a gesture lexicon (i.e. all the gestures that are shared between speakers) as well as speaker style (i.e., a statistical model which captured the individual differences in the use and frequency of each gestures) from a manually annotated video corpus. Their system was then able to produce gestures for any arbitrary text input in the style of a particular speaker, though the text still had to be annotated (i.e., provide additional information on word boundaries, utterances, and information structure and focus of each utterance). Similarly, the model proposed by Bergmann and Kopp [12] could learn idiosyncratic features (i.e., features that are characteristic of an individual speaker) from human communicative data. In addition, they also modelled more universal gesture features (such as shape/gesture form) defined by a set of rules.

Often gesture production systems in embodied agents use gesture templates which lead to almost identical gestures. For instance, Ng-hing, Luo, Okita [108] created a gesture lexicon based on the analysis of video corpora where gesture phases (start, stroke, retraction) were annotated manually. However, human gestures are also highly variable in terms of their trajectory (i.e. the shape of gesture) and such a templatebased approach again is highly deterministic. Other systems have attempted to produce gestures on the fly which (in principle) may add variability. For instance, in the Kopp, Bergmann, Wachsmuth system [64] gesture form was not fixed, but produced from multimodal representations of objects, locations and their spatial relations. More recently, Ferstl and McDonnel [35] used a recurrent neural network to produce gesture motion directly from prosodic speech features. Interestingly, the network was first pre-trained with a motion modelling task before training the final speech-to-gesture model. A similar approach was taken in Ferstl, Neff, McDonnell [36], where generative adversarial networks were used for producing gestures from input speech.

Finally, to be effective and believable, iconic gestures and points must be tightly linked to and perfectly time-aligned with the accompanied speech. The BEAT system of Cassell et al. [23] uses word and phoneme timings to construct a multimodal animation schedule. Whereas the system of Kopp and Wachsmuth [65], coordinates gestures and speech based on the assumption that both speech and gesture could be divided into successive, single units. More specifically, gesture motion is divided into gesture units (e.g. preparation, stroke, retraction and hold) while speech into intonation units (e.g. intonation phases with exactly one primary pitch accent, that is, nucleus). Gesture units then are aligned with speech units in a way to allow the gesture stroke to start before the affiliated word (or nucleus) and lasts throughout. To maintain the 
synchrony between the gesture and speech, the gesture is adapted to the structure and timing of accompanied speech.

Gaze has also been implemented on virtual agents and robots. Some models used heuristics derived from the analysis of human dyadic conversations. For instance, Cassell et al. [22] analyzed the distributions of gaze and head movements. They found that speakers look away from the listener at the beginning of a theme (what is talked about, the topic, with 0.70 probability) and look toward the listener at the beginning of a rheme (what it is said about the topic, with 0.73 probability). Mutlu et al. [79] extended this empirical model with parameters derived from the analysis of data collected from a professional storyteller. More specifically, they first determined main gaze locations. Then, they derived probabilities of looking towards and looking away from the listener from the analysis of the frequencies of the storyteller's gaze at each location. The gaze durations followed a normal distribution with the mean and standard deviation values of the storyteller's gaze. Similar approach was used by Mutlu et al. [80], where the analysis of empirical studies directly informed the gaze mechanisms of a humanoid robot. In addition to previous works, this model also implemented topic-signaling mechanism: for each new topic, the robot produced a series of gaze shifts based on the gaze patterns identified for each conversational scenario.

Several models produce head and gaze movements based on prosodic features extracted from speech signal. For instance, in the model of Albrecht, Haber and Seidel [3], the head and eyebrow raise depending on the value of pitch, while gaze directed at an immobile, fixed location during pauses for thinking and word search, and random movement are produced during normal speech. Lee, Ma, Deng [69] also proposed a system that produces head motion, gaze, and eyelid motion simultaneously based on speech input. Separate statistical model for each behavior were learned from data collected during dyadic face-to-face conversation and single subject's speaking scenario. More believable performance of a virtual agent can be achieved by producing behaviors based on semantics in addition to prosody. For instance, Marsella et al. [72] system produced head movements and gaze (as well as other multimodal cues) from the prosodic and semantic analysis of the speech. The system is based on a set of rules derived from psychological literature, but also based on data collected from human face-to-face interactions which were annotated and analyzed to extract the dynamics of multimodal cues.

Another important cue, mouth movement, makes the communication with an embodied agent more natural and believable. The existing systems produce mouth animations from either natural speech or a text-to-speech engine. Often mouth animation is done by first creating a canonical set of mouth shapes (i.e. visemes) that map one or more phoneme to a corresponding viseme, and later on, given the sequence of visemes, by interpolating the visemes to animate the mouth. However, such an approach leads to poor results due to discontinuities in the sequences of mouth movements. Better results are obtained taking into account coarticulation, where the shape of the current viseme is affected by adjacent phonemes. For instance, Cohen and Massaro [26] modeled coarticulation using dominance functions, which for each speech unit described the influence of its preceding and following viseme. Some approaches use statistical models to learn the co-articulation. For instance, Ding and Pelachaud's system [30] takes 
as an input a spoken text decomposed into phonemes and their durations. Gaussian Mixture Models (GMM) are built to infer the shape of mouth for each phoneme. For producing smooth mouth animation, the HMM interpolations are used as interpolation function. Similarly, Luo, Yu, Wang [70], used GMM for producing mouth movements from speech. They incorporated previous visual features into the model which helped to eliminate the discontinuities in the mouth movement. Interestingly, Xu et al. [119] showed that lip animation can be achieved using only a canonical set of phoneme pairs. More recent approaches use machine learning for lip movement production. For instance, Aneja and Li [7] proposed a deep learning system to automatically produce from input audio mouth movements (i.e. viseme sequences) for 2D virtual characters.

Embodied agents use text-to-speech (TTS) systems to produce speech. For the speech to sound more natural (be more variable yet consistent), TTS systems incorporate prosody models to produce prosodic properties of speech (i.e. pitch, intonation, duration, stress and style). Traditionally, these systems focused on predicting phoneme duration, and later on predicting fundamental frequency (F0) $[104,106])$. Recent TTS systems, represent prosody implicitly within a neural network [90, 113]. Although these speech synthesis models produce natural prosody and expression, it is unclear how they can produce more variable, hence more humanlike, prosodic features. Here several studies focused on modelling emotions in speech $[112,113]$ as well as the different ways in which the same text can be said as well as modelling. For instance, Wang's and colleagues [113] goal was to provide the systems with the capability to choose a speaking style appropriate for the given context, moving beyond modelling the naturalness of speech. Similarly, Wan et al. [112] presented a new system able to produce more variations in produced synthetic speech.

It is important to note that, again, the focus is predominantly on single (e.g. iconic gestures or gaze), rather than on combined cues. Only a few attempts considered the generation of more than one multimodal cue. For instance, Salem et al. [99] looked at both gaze and gestures that accompany speech. The complexity of robot's gaze behavior, however, was reduced to directional looks (i.e. looking right when pointing right). A more complex multimodal interaction was attempted by Huang and Mutlu [52] who applied a learning-based approach to model how humans coordinate speech, gaze, and gestures during storytelling. The alignment among these cues was modelled using a dynamic Bayesian network that learned the distribution and alignment parameters automatically from annotated data from a human multimodal corpus.

\subsection{Summary and Way Forward: Mutual benefits from studies on multimodal communication}

From a cognitive neuroscience/psychology perspective, a main goal is to understand communication and its neural circuitry in humans. From an AI perspective, a main goal is to develop embodied agents that can communicate with humans in the most effective manner. In both camps, the investigation of multimodal communication is a very recent development but as it clear from the previous sections, a promising one. A growing literature is emerging showing that looking at human communication from a multimodal perspective is providing novel insights into our understanding of the psychological and neural mechanisms $[49,110]$ and that agents that use multimodal cues 
have a number of benefits over agents that only use speech such as being perceived as better communication partners and improving the effectiveness of the communication.

However, there are a number of general issues that at the moment preclude faster development of this area. The most important and general one is perhaps that in both camps much of the existing work has considered only how to integrate one of the multimodal cues (e.g., either gesture, or gaze) at a time with speech, rather than considering the joint contribution of the different multimodal cues to production or comprehension. This is limiting the potential understanding of the phenomenon as human communication is characterized by speech accompanied by all the multimodal cues we have discussed here (see e.g., Zhang et al., in prep). Considering a single cue at a time, may also render the problem of recognition and production by embodied agents harder. This is because the different multimodal cues are correlated among one another and correlated with speech, hence they can provide additional constraints (e.g., as discussed above, the referent of a point can be better disambiguated using speech combined with gaze [51]). Thus, approaches that attempt to understand and use the different multimodal cues at the same time may be the most promising way forward.

\section{Development and Coding of Shared Corpora}

As already mentioned, a major bottleneck is the lack of data on (i) when and which of the multimodal cues are used across topics and across speakers; (ii) how the cues are coordinated - how they are correlated - in face-to-face communication. For example, words accompanied by gestures tend to be marked by prosodic stress [49], and increased prosodic contour is correlated with larger mouth movements. In order to answer these questions, corpora in which each cue is annotated and time-aligned with the speech are needed. This is really hard. From naturalistic face-to-face interactions, the different cues need to be extracted and then coded. The current practice is to hand-code a number of these cues (e.g., type of gestures) and then to time-lock the coded cues along with segmented speech. Even for those cues that can be automatically extracted from video (e.g., gaze) or audio (e.g., prosodic contour), manual intervention is still often necessary (e.g., for gaze, the objects being looked at often need to be handcoded); or desirable (e.g., for prosody where automatic methods still lack high levels of precision). For mouth movements as well as gestures, although automatic systems, in principle, can extract motion patterns in a quite reliable manner (especially if speakers wear motion markers), these need then to be interpreted in order to be usable. Thus, coded and time-aligned data is still very expensive.

Collaborations among psychologists and computer scientists bringing together expertise in designing behavioural experiments with state-of-the-art sensing and ML technology in the development of corpora of face-to-face communication can offer a way out of the empasse created by manual coding.

\section{Toward a mechanistic understanding of multimodal communication}

Building embodied agents that use multimodal cues in a human-like manner calls for a detailed understanding of how these work in humans. On the other hand, developing 
computational models (which can be implemented in embodied agents) of humanlike multimodal language processing and production provides cognitive scientists with explicit mechanistic accounts of the processes that can be tested. There is a longstanding tradition in cognitive science and neuroscience in using computational models to provide explicit descriptions of cognitive processes (e.g., [91, 101, 78]). Especially for complex problems, like multimodal communication, such an approach promises to provide novel and important insights.

It has been argued that in multimodal communication, signals are processed at different levels in a predictive manner [49]. For example, seeing a speaker's lips shaped to produce a $[\mathrm{w}]$ sound may restrict the search space for predictions about upcoming words to a phonetically congruent set of candidates. This candidate set may be pruned further by other co-occurring signals both in the visual modality, such as raised eyebrows and a lifted palm-up open hand, and in the auditory modality, such as raising pitch. Such co-occurring multimodal combinations of signals would then trigger the prediction of a question being produced. This prediction, in turn, could then feed downwards, increasing the expectation for a "wh" word being uttered, plus a questiontypical syntactical structure, and so forth. Implementing such prediction-based mechanisms in computational models (or embodied agents) provides the most powerful way to assess their plausibility as an account of human multimodal communication (see [91] for an example).

\section{Studying human communication with embodied agents}

Language has been studied predominantly in its unimodal instantiation as speech or text. This has been done primarily for experimental control reasons. Recent developments in virtual technology and humanoid robotics, provide a way of studying human language processing in a controlled, yet, naturalistic face-to-face setting. Importantly, both VR and humanoid robots provide a good balance between experimental control and ecological validity $[86,89,116]$. Using robots and virtual avatars as experimental stimuli (i.e. conversational partners) ensures consistency and replicability of their behaviors between participants, and hence, the reproducibility of the results. It also allows (to a certain degree) to study communication in a social interaction setting, instead of often used "observational" paradigms. Moreover, the behavior of robots and virtual agents, can be controlled with relatively high precision, allowing to investigate questions that would not be possible using more traditional methods. One such problem could be how the timing of different multimodal cues affects language processing. Using, for instance, an avatar we can manipulate separately the different cues (gestures, gaze, prosody etc.) and in a controlled way change their presence/absence as well as their relative timings. This would not be possible without virtual agents or humanoid robots.

In addition, embodied agents can be used as a test-bed for testing computational models (e.g. [29, 77, 81]). This is particularly important when we consider the multimodal nature of language, that utilizes a wide range of different cues, and hence, different articulators. Computational models can be designed following the insights from empirical studies in humans, and subsequently validated on an agent that, similar to humans, interacts with its physical and social environment through its body. 
The results from such computational studies can, in turn, lead to revision of the models but also, more generally, to a better understanding of underpinning mechanisms of human communication.

Acknowledgements. When writing this paper, the authors were supported by European Research Council Advanced Grant (ECOLANG, 743035) to GV. GV was further supported by a Royal Society Wolfson Research Merit Award (WRM370016). 


\section{Bibliography}

[1] L. P. Acredolo and S. Goodwyn. "Symbolic gesturing in normal infants". In: Child Development 59.2 (1988), pp. 450-466.

[2] H. Admoni, C. Datsikas, and B. Scassellati. "Speech and Gaze Conflicts in Collaborative Human-Robot Interactions". In: Proceedings of the Annual Meeting of the Cognitive Science Society. 2014.

[3] I. Albrecht, J. Haber, and H. Seidel. "Automatic Generation of Non-Verbal Facial Expressions from Speech". In: Advances in Modelling, Animation and Rendering. Ed. by J. Vince and R. Earnshaw. 2002.

[4] M. W. Alibali et al. "Teachers' gestures and speech in mathematics lessons: forging common ground by resolving trouble spots". In: ZDM 45 (2013), pp. 425440.

[5] G. L. Allen. "Gestures Accompanying Verbal Route Directions: Do They Point to a New Avenue for Examining Spatial Representations?" In: Spatial Cognition 83 Computation 3.4 (2003), pp. 259-268.

[6] S. Andrist et al. "Conversational gaze aversion for humanlike robots". In: Proceedings of the 2014 ACM/IEEE international conference on Human-robot interaction. 2014.

[7] D. Aneja and W. Li. "Real-Time Lip Sync for Live 2D Animation". In: (2019). URL: arXiv: 1910.08685.

[8] J. Ang et al. "Prosody-based automatic detection of annoyance and frustration in human-computer dialog". In: INTERSPEECH. 2002.

[9] P. Arnold and F. Hill. "Bisensory augmentation: a speechreading advantage when speech is clearly audible and intact". In: British Journal of Psychology (London, England: 1953) 92.Pt 2 (2001), pp. 339-355.

[10] D. A. Baldwin. "Early referential understanding: Infants' ability to recognize referential acts for what they are". In: Developmental Psychology 29.5 (1993), pp. 832-843.

[11] P. Barros et al. "Real-time gesture recognition using a humanoid robot with a deep neural architecture". In: 2014 IEEE-RAS International Conference on Humanoid Robots. 2014.

[12] K. Bergmann and S. Kopp. "GNetIc - Using Bayesian Decision Networks for Iconic Gesture Generation". In: Intelligent Virtual Agents. Ed. by Z. Ruttkay et al. Lecture Notes in Computer Science. Springer, 2009, pp. 76-89.

[13] K. Bergmann, S. Kopp, and F. Eyssel. "Individualized Gesturing Outperforms Average Gesturing - Evaluating Gesture Production in Virtual Humans". In: Intelligent Virtual Agents. Ed. by J. Allbeck et al. Lecture Notes in Computer Science. Berlin, Heidelberg: Springer, 2010, pp. 104-117. 
[14] D. Le M. Bolinger. Degree words. Paris; The Hague: Mouton, 1972.

[15] J.-D. Boucher et al. "I Reach Faster When I See You Look: Gaze Effects in Human-Human and Human-Robot Face-to-Face Cooperation". In: Frontiers in Neurorobotics 6 (2012).

[16] C. Breazeal et al. "Effects of nonverbal communication on efficiency and robustness in human-robot teamwork". In: 2005 IEEE/RSJ International Conference on Intelligent Robots and Systems. 2005.

[17] C. Bregler and Y. Konig. "'Eigenlips" for robust speech recognition". In: Proceedings of ICASSP '94. IEEE International Conference on Acoustics, Speech and Signal Processing. Apr. 1994.

[18] R. Brooks and A. N. Meltzoff. "The development of gaze following and its relation to language". In: Developmental Science 8.6 (2005), pp. 535-543.

[19] S. Buisine and J.-C. Martin. "The effects of speech-gesture cooperation in animated agents' behavior in multimedia presentations". In: Interacting with Computers 19.4 (2007), pp. 484-493.

[20] D. Burnham and B. Dodd. "Auditory-visual speech integration by prelinguistic infants: perception of an emergent consonant in the McGurk effect". In: Developmental Psychobiology 45.4 (2004), pp. 204-220.

[21] A. Cangelosi and T. Ogata. "Speech and Language in Humanoid Robots". In: Humanoid Robotics: A Reference. Ed. by Ambarish Goswami and Prahlad Vadakkepat. Springer Netherlands, 2016, pp. 1-32. ISBN: 978-94-007-7194-9. DOI: $10.1007 / 978-94-007-7194-9 \_135-1$.

[22] J. Cassell et al. "Animated conversation: rule-based generation of facial expression, gesture \& spoken intonation for multiple conversational agents". In: Proceedings of the 21st annual conference on Computer graphics and interactive techniques - SIGGRAPH' '94. 1994.

[23] J Cassell et al. "More than just a pretty face: conversational protocols and the affordances of embodiment". In: Knowledge-Based Systems 14.1 (2001), pp. 5564 .

[24] J. C. Castillo et al. "Dynamic Gesture Recognition for Social Robots". In: Social Robotics. Ed. by A. Kheddar et al. Vol. 10652. Cham: Springer International Publishing, 2017, pp. 495-505.

[25] C. Chandrasekaran et al. "The Natural Statistics of Audiovisual Speech". In: PLOS Computational Biology 5.7 (2009), e1000436.

[26] M. M. Cohen and D. W. Massaro. "Modeling Coarticulation in Synthetic Visual Speech". In: Models and Techniques in Computer Animation. Ed. by N. M. Thalmann and D. Thalmann. Computer Animation Series. Tokyo: Springer Japan, 1993, pp. 139-156.

[27] N. Cooke, A. Shen, and M. Russell. "Exploiting a 'gaze-Lombard effect' to improve ASR performance in acoustically noisy settings". In: 2014 IEEE International Conference on Acoustics, Speech and Signal Processing (ICASSP). 2014.

[28] A. Cutler, D. Dahan, and W. van Donselaar. "Prosody in the Comprehension of Spoken Language: A Literature Review". In: Language and Speech (1997). 
[29] A. Di Nuovo and J. L. McClelland. "Developing the knowledge of number digits in a child-like robot". In: Nature Machine Intelligence 1.12 (2019), pp. 594-605.

[30] Y. Ding and C. Pelachaud. "Lip animation synthesis: a unified framework for speaking and laughing virtual agent". In: AVSP. 2015.

[31] M. W. Doniec, G. Sun, and B. Scassellati. "Active Learning of Joint Attention". In: 2006 6th IEEE-RAS International Conference on Humanoid Robots. 2006.

[32] Drijvers, L. and Özyürek, A. "Visual Context Enhanced: The Joint Contribution of Iconic Gestures and Visible Speech to Degraded Speech Comprehension". In: Journal of Speech, Language, and Hearing Research 60.1 (2017), pp. $212-222$.

[33] D. Droeschel, J. Stückler, and S. Behnke. "Learning to interpret pointing gestures with a time-of-flight camera". In: 2011 6th ACM/IEEE International Conference on Human-Robot Interaction (HRI). 2011.

[34] A. Fernald and T. Simon. "Expanded intonation contours in mothers' speech to newborns". In: Developmental Psychology 20.1 (1984), pp. 104-113.

[35] Y. Ferstl and R. McDonnell. "Investigating the use of recurrent motion modelling for speech gesture generation". In: Proceedings of the 18th International Conference on Intelligent Virtual Agents. 2018.

[36] Y. Ferstl, M. Neff, and R. McDonnell. "Multi-objective adversarial gesture generation". In: Motion, Interaction and Games. 2019.

[37] D. Formolo and T. Bosse. "A Conversational Agent that Reacts to Vocal Signals". In: Intelligent Technologies for Interactive Entertainment. 8th International Conference. 2016.

[38] L. A. Friedman. On the other hand: new perspectives on American sign language. Academic Press, 1977.

[39] T. Fu et al. "Integrating prosodic information into recurrent neural network language model for speech recognition". In: 2015 Asia-Pacific Signal and Information Processing Association Annual Summit and Conference (APSIPA). 2015 .

[40] S. Goldin-Meadow. "The role of gesture in communication and thinking". In: Trends in Cognitive Sciences 3.11 (1999), pp. 419-429.

[41] S. Goldin-Meadow et al. "Explaining math: gesturing lightens the load". In: Psychological Science 12.6 (2001), pp. 516-522.

[42] S. Grassmann and M. Tomasello. "Two-year-olds use primary sentence accent to learn new words". In: Journal of Child Language 34.3 (2007), pp. 677-687.

[43] Z. M. Griffin and K. Bock. "What the eyes say about speaking". In: Psychological Science 11.4 (2000), pp. 274-279.

[44] J. E. Hanna and S. E. Brennan. "Speakers' eye gaze disambiguates referring expressions early during face-to-face conversation". In: Journal of Memory and Language 57.4 (2007), pp. 596-615.

[45] K. Hawthorne and L.A. Gerken. "From pauses to clauses: prosody facilitates learning of syntactic constituency". In: Cognition 133.2 (2014), pp. 420-428. 
[46] D. S. Herold, L. C. Nygaard, and L. L. Namy. "Say it like you mean it: mothers' use of prosody to convey word meaning". In: Language and Speech 55.Pt 3 (2012), pp. 423-436.

[47] D. Heylen et al. "Experimenting with the Gaze of a Conversational Agent". In: Proceedings of the International CLASS Workshop on Natural, Intelligent and Effective Interaction in Multimodal Dialogue Systems. Copenhagen. 2002.

[48] H. Holle and T. C. Gunter. "The role of iconic gestures in speech disambiguation: ERP evidence". In: Journal of Cognitive Neuroscience 19.7 (2007), pp. $1175-1192$.

[49] J. Holler and S. C. Levinson. "Multimodal Language Processing in Human Communication". In: Trends in Cognitive Sciences 23.8 (2019), pp. 639-652.

[50] J. Holler et al. "Social eye gaze modulates processing of speech and co-speech gesture". In: Cognition 133.3 (2014), pp. 692-697.

[51] H. Holzapfel, K. Nickel, and R. Stiefelhagen. "Implementation and evaluation of a constraint-based multimodal fusion system for speech and 3D pointing gestures". In: Proceedings of the 6th international conference on Multimodal interfaces. 2004.

[52] C.-M. Huang and . Mutlu. "Modeling and Evaluating Narrative Gestures for Humanlike Robots". In: Robotics: Science and Systems. 2013.

[53] T. Iio et al. "Investigating Entrainment of People's Pointing Gestures by Robot's Gestures Using a WOZ Method". In: International Journal of Social Robotics 3.4 (2011), pp. 405-414.

[54] S. Ivaldi et al. "Robot initiative in a team learning task increases the rhythm of interaction but not the perceived engagement". In: Front. Neurorobot. (2014).

[55] J. M. Iverson et al. "Gesturing in mother-child interactions". In: Cognitive Development 14.1 (1999), pp. 57-75.

[56] D. E. Karreman et al. "What happens when a robot favors someone? How a tour guide robot uses gaze behavior to address multiple persons while storytelling about art". In: Proceedings of the 8th ACM/IEEE international conference on Human-robot interaction. 2013.

[57] S. D. Kelly, A. Ozyürek, and E. Maris. "Two sides of the same coin: speech and gesture mutually interact to enhance comprehension". In: Psychological Science 21.2 (2010), pp. 260-267.

[58] S. Kettebekov, M. Yeasin, and R. Sharma. "Improving continuous gesture recognition with spoken prosody". In: 2003 IEEE Computer Society Conference on Computer Vision and Pattern Recognition. 2003.

[59] M. Kipp et al. "Towards Natural Gesture Synthesis: Evaluating Gesture Units in a Data-Driven Approach to Gesture Synthesis". In: ed. by Catherine Pelachaud et al. Lecture Notes in Computer Science. Berlin, Heidelberg: Springer, 2007, pp. $15-28$.

[60] S. Kita. "How representational gestures help speaking". In: Language and Gesture. Ed. by D. McNeill. Cambridge University Press, 2000, pp. 162-185. 
[61] S. Kita, M. W. Alibali, and M. Chu. "How do gestures influence thinking and speaking? The gesture-for-conceptualization hypothesis". In: Psychological Review 124.3 (2017), pp. 245-266.

[62] D. B. Koons and C. J. Sparrell. "Iconic: speech and depictive gestures at the human-machine interface". In: Conference Companion on Human Factors in Computing Systems. Apr. 1994.

[63] D. B. Koons, C. J. Sparrell, and K. R. Thorisson. "Integrating simultaneous input from speech, gaze, and hand gestures". In: Intelligent multimedia interfaces. USA: American Association for Artificial Intelligence, 1993, pp. 257-276.

[64] S. Kopp, K. Bergmann, and I. Wachsmuth. "Multimodal Communication from Multimodal Thinking - towards an Integrated Model of Speech and Gesture Production". In: Int. J. Semantic Computing 2 (2008), pp. 115-136.

[65] S. Kopp and I. Wachsmuth. "Synthesizing multimodal utterances for conversational agents". In: Computer Animation and Virtual Worlds 15.1 (2004), pp. $39-52$.

[66] N. C. Krämer, N. Simons, and S. Kopp. "The Effects of an Embodied Conversational Agent's Nonverbal Behavior on User's Evaluation and Behavioral Mimicry". In: IVA. 2007.

[67] R. M. Krauss, Y. Chen, and R. F. Gottesman. "Language and Gesture: Lexical gestures and lexical access: a process model". In: Language and Gesture. Cambridge University Press, 2000. IsBN: 978-0-511-62085-0.

[68] E. Kushnerenko et al. "Electrophysiological evidence of illusory audiovisual speech percept in human infants". In: Proceedings of the National Academy of Sciences of the United States of America 105.32 (2008), pp. 11442-11445.

[69] B. H. Le, X. Ma, and Z. Deng. "Live Speech Driven Head-and-Eye Motion Generators". In: IEEE Transactions on Visualization and Computer Graphics 18.11 (2012), pp. 1902-1914.

[70] C. Luo, J. Yu, and Z. Wang. "Synthesizing real-time speech-driven facial animation". In: 2014 IEEE International Conference on Acoustics, Speech and Signal Processing (ICASSP). 2014.

[71] W. Ma et al. "Word Learning in Infant- and Adult-Directed Speech". In: Language Learning and Development 7.3 (2011), pp. 185-201.

[72] S. Marsella et al. "Virtual character performance from speech". In: Proceedings of the 12th ACM SIGGRAPH/Eurographics Symposium on Computer Animation. 2013.

[73] H. McBreen and M. A. Jack. Empirical Evaluation of Animated Agents in a Multi-Modal E-Retail Application. Tech. rep. 2000.

[74] Harry Mcgurk and John Macdonald. "Hearing lips and seeing voices". In: Nature 264.5588 (1976), pp. 746-748.

[75] M. Morales, P. Mundy, and J. Rojas. "Following the direction of gaze and language development in 6-month-olds". In: Infant Behavior 83 Development 21.2 (1998), pp. 373-377. 
[76] L.-P. Morency, C. M. Christoudias, and T. Darrell. "Recognizing gaze aversion gestures in embodied conversational discourse". In: Proceedings of the 8th international conference on Multimodal interfaces. 2006.

[77] A. F. Morse et al. "Posture Affects How Robots and Infants Map Words to Objects". In: PLOS ONE 10.3 (2015), e0116012.

[78] Y. Munakata et al. "Rethinking infant knowledge: Toward an adaptive process account of successes and failures in object permanence tasks". In: Psychological Review 104.4 (1997), pp. 686-713.

[79] B. Mutlu, J. Forlizzi, and J. Hodgins. "A Storytelling Robot: Modeling and Evaluation of Human-like Gaze Behavior". In: 2006 6th IEEE-RAS International Conference on Humanoid Robots. 2006.

[80] B. Mutlu et al. "Conversational gaze mechanisms for humanlike robots". In: ACM Transactions on Interactive Intelligent Systems (TiiS) 1.2 (2012), 12:112:33.

[81] Y. Nagai. "Predictive learning: its key role in early cognitive development". In: Philosophical Transactions of the Royal Society B: Biological Sciences 374.1771 (2019), p. 20180030.

[82] K. Nickel and R. Stiefelhagen. "Real-Time Recognition of 3D-Pointing Gestures for Human-Machine-Interaction". In: Pattern Recognition. Ed. by B. Michaelis and G. Krell. Lecture Notes in Computer Science. Berlin, Heidelberg: Springer, 2003, pp. 557-565.

[83] K. Noda et al. "Audio-visual speech recognition using deep learning". In: Applied Intelligence 42.4 (2015), pp. 722-737.

[84] S. Ozçalişkan and S. Goldin-Meadow. "Gesture is at the cutting edge of early language development". In: Cognition 96.3 (2005), B101-113.

[85] O. Palinko et al. "Robot reading human gaze: Why eye tracking is better than head tracking for human-robot collaboration". In: 2016 IEEE/RSJ International Conference on Intelligent Robots and Systems (IROS). 2016.

[86] X. Pan and A. F. de C. Hamilton. "Why and how to use virtual reality to study human social interaction: The challenges of exploring a new research landscape". In: British Journal of Psychology 109.3 (2018), pp. 395-417.

[87] M. Paulus and P. Fikkert. "Conflicting Social Cues: Fourteen-and 24-MonthOld Infants' Reliance on Gaze and Pointing Cues in Word Learning". In: Journal of Cognition and Development 15.1 (2014), pp. 43-59.

[88] Jonathan E. Peelle and Mitchell S. Sommers. "Prediction and constraint in audiovisual speech perception". In: Cortex; a Journal Devoted to the Study of the Nervous System and Behavior 68 (2015), pp. 169-181.

[89] D. Peeters. "Virtual reality: A game-changing method for the language sciences". In: Psychonomic Bulletin 83 Review 26.3 (2019), pp. 894-900.

[90] W. Ping et al. "Deep Voice 3: Scaling Text-to-Speech with Convolutional Sequence Learning". In: (2018). URL: arXiv:1710.07654.

[91] M. Rabovsky, S. S. Hansen, and J. L. McClelland. "Modelling the N400 brain potential as change in a probabilistic representation of meaning". In: Nature Human Behaviour 2.9 (2018), pp. 693-705. 
[92] F. H. Rauscher, R. M. Krauss, and Y. Chen. "Gesture, Speech, and Lexical Access: The Role of Lexical Movements in Speech Production". In: Psychological Science 7.4 (1996), pp. 226-231.

[93] L. D. Riek et al. "Cooperative gestures: Effective signaling for humanoid robots". In: 2010 5th ACM/IEEE International Conference on Human-Robot Interaction (HRI). 2010.

[94] K. J. Rohlfing. "Meaning in the objects". In: Experimental Pragmatics/Semantics (2011).

[95] F. de Rosis et al. "'You are Sooo Cool, Valentina!' Recognizing Social Attitude in Speech-Based Dialogues with an ECA". In: Affective Computing and Intelligent Interaction. Ed. by A. C. R. Paiva, R. Prada, and R. W. Picard. Lecture Notes in Computer Science. Berlin, Heidelberg: Springer, 2007, pp. 179-190.

[96] M. L. Rowe and S. Goldin-Meadow. "Early gesture selectively predicts later language learning". In: Developmental science 12.1 (2009), pp. 182-187.

[97] M. L. Rowe, Ş. Özçalişkan, and S. Goldin-Meadow. "Learning words by hand: Gesture's role in predicting vocabulary development". In: First language 28.2 (2008), pp. 182-199.

[98] J. de Ruiter. "The production of gesture and speech". In: Language and Gesture. 2000.

[99] M. Salem et al. "A friendly gesture: Investigating the effect of multimodal robot behavior in human-robot interaction". In: 2011 RO-MAN. 2011.

[100] M. Salem et al. "To err is human(-like): Effects of robot gesture on perceived anthropomorphism and likability". In: International Journal of Social Robotics 5.3 (2013), pp. 313-323.

[101] M. S. Seidenberg and J. L. McClelland. "A distributed, developmental model of word recognition and naming". In: Psychological Review 96.4 (1989), pp. 523568.

[102] A. Showers and M. Si. "Pointing Estimation for Human-Robot Interaction Using Hand Pose, Verbal Cues, and Confidence Heuristics". en. In: Social Computing and Social Media. Technologies and Analytics. Ed. by G. Meiselwitz. Lecture Notes in Computer Science. Springer International Publishing, 2018, pp. 403-412.

[103] T. Sowa and I. Wachsmuth. "Interpretation of Shape-Related Iconic Gestures in Virtual Environments". In: Gesture and Sign Language in Human-Computer Interaction. Ed. by G. Goos et al. Vol. 2298. Berlin, Heidelberg: Springer Berlin Heidelberg, 2002, pp. 21-33.

[104] D. Stanton, Y. Wang, and RJ Skerry-Ryan. "Predicting Expressive Speaking Style from Text in End-To-End Speech Synthesis". In: 2018 IEEE Spoken Language Technology Workshop (SLT). 2018.

[105] M. Staudte and M. W. Crocker. "Investigating joint attention mechanisms through spoken human-robot interaction". In: Cognition 120.2 (2011), pp. 268291.

[106] Paul Taylor, Alan W. Black, and Richard Caley. "The architecture of the Festival speech synthesis system". In: $S S W .1998$. 
[107] E. D. Thiessen, E. A. Hill, and J. R. Saffran. "Infant-Directed Speech Facilitates Word Segmentation". In: Infancy 7.1 (2005), pp. 53-71.

[108] V. Ng-Thow-Hing, L. Pengcheng, and S. Okita. "Synchronized gesture and speech production for humanoid robots". In: 2010 IEEE/RSJ International Conference on Intelligent Robots and Systems. 2010.

[109] M. Tomasello. Constructing a language: A usage-based theory of language acquisition. Cambridge, MA, US: Harvard University Press, 2003.

[110] G. Vigliocco, P. Perniss, and D. Vinson. "Language as a multimodal phenomenon: implications for language learning, processing and evolution". In: Philosophical Transactions of the Royal Society B: Biological Sciences 369.1651 (2014).

[111] G. Vigliocco et al. "Onomatopoeia, gestures, actions and words: how do caregivers use multimodal cues in their communication to children?" In: Proceedings of the 41th Annual Meeting of the Cognitive Science Society. 2019.

[112] V. Wan et al. "CHiVE: Varying Prosody in Speech Synthesis with a Linguistically Driven Dynamic Hierarchical Conditional Variational Network". In: 2019. URL: arXiv: 1905.07195.

[113] Y. Wang et al. "Style Tokens: Unsupervised Style Modeling, Control and Transfer in End-to-End Speech Synthesis". In: 2018. URL: arXiv:1803.09017.

[114] V. van Wassenhove, K. W. Grant, and D. Poeppel. "Visual speech speeds up the neural processing of auditory speech". In: Proceedings of the National Academy of Sciences of the United States of America 102.4 (2005), pp. 1181-1186.

[115] S. R. Waxman and J. L. Lidz. "Early Word Learning". In: Handbook of child psychology: Cognition, perception, and language, Vol. 2, 6th ed. Hoboken, NJ, US: John Wiley \& Sons Inc, 2006, pp. 299-335.

[116] A. Wykowska, T. Chaminade, and G. Cheng. "Embodied artificial agents for understanding human social cognition". In: Philosophical Transactions of the Royal Society of London. Series B, Biological Sciences 371.1693 (2016).

[117] Y. Xiao et al. "Human-robot interaction by understanding upper body gestures". In: Presence: Teleoperators and Virtual Environments 23.2 (2014), pp. 133154.

[118] T. L. Xu, H. Zhang, and C. Yu. "See You See Me: the Role of Eye Contact in Multimodal Human-Robot Interaction". In: ACM transactions on interactive intelligent systems 6.1 (2016).

[119] Y. Xu et al. "A Practical and Configurable Lip Sync Method for Games". In: Proceedings of Motion on Games. 2013.

[120] C. Yu, P. Schermerhorn, and M. Scheutz. "Adaptive eye gaze patterns in interactions with human and artificial agents". In: ACM Transactions on Interactive Intelligent Systems (TiiS) 1.2 (2012), 13:1-13:25. 